\title{
Determination of the Electron Thermal Diffusivity at WEGA Stellarator
}

\author{
D. ZHANG, M. GLAUBITZ, H.P. LAQUA, S. MARSEN, M. OTTE, T. STANGE \\ Max-Planck-Institut für Plasmaphysik, EURATOM Ass., D-17491 Greifswald, Germany
}

\begin{abstract}
For determining the thermal diffusivity in WEGA the electron cyclotron resonance heating (ECRH) power from a $28 \mathrm{GHz}$ gyrotron is square-wave modulated to provide a periodically varying energy source in the plasma center causing a modulation mainly of the electron temperature $T_{e}$. A fast Si-diode bolometer system, whose 16-channels view the entire plasma cross-section, is employed to monitor the heat propagation process. A Fast Fourier Transform (FFT) analysis shows clear coherency of the line-integrated signals among different channels and monotonic radial increment of the phase delays of the fundamental components in the central channel signals, allowing the determination of the electron thermal diffusivity coefficient D. A purely diffusive heat transport is assumed and is simulated using a Monte Carlo method. The thermal diffusivity is determined by matching the simulated results to the measured ones. A typical value of $D=1.9 \mathrm{~m}^{2} / \mathrm{s}$ is obtained. This value is then compared with the result based on a local power balance analysis. Within the error bars the local power balance calculation yields a similar diffusivity value. The limitations and conditions of utilizing bolometric diagnostic for this purpose are discussed.
\end{abstract}

\section{Introduction}

Electron thermal conductivity is one of the most important parameters characterizing the confinement performance of a fusion device [1-4]. A centrally-localized, modulated ECRheating power deposition makes a thermal transport study possible. By careful choices of the modulation frequency and amplitude, a varying ECR-heating power causes a perturbation prominently of the electron temperature $T_{e}$. The propagation of the perturbation is closely related to a heat transport process. There are different diagnostics capable of measuring this temperature response, e.g. the electron cyclotron emission (ECE) diagnostic $[1,3]$ and the soft $\mathrm{X}$-ray array [2]. In this paper, however, we employ a fast bolometer system at WEGA for this purpose.

WEGA [5] is a medium-sized classical stellarator ( $R=72 \mathrm{~cm}, a=0.19 \mathrm{~cm}, l=2, m=$ 5) operated in IPP-Greifswald. The plasma temperature at WEGA $\left(T_{e}<60 \mathrm{eV}\right)$ is close to that at the edge region in $\mathrm{W} 7-\mathrm{X}$; and at its own edge region $T_{e}$ is below $5 \mathrm{eV}$. No evident impurities coming from plasma facing components have been observed in the confined plasmas with $\mathrm{He}, \mathrm{Ar}$, and $\mathrm{H}_{2}$ as working gases. This provides an opportunity to study basic physics in quasi-pure magnetically confined plasmas. The bolometer diagnostic employs two kinds of detectors, i.e. Au-resistive type based on a Kapton foil substrate [6] and Siphotodiode array of a type AXUV-16EL/G [7]. They have been installed to monitor the plasma radiation features associated with different microwave heating scenarios, and at the same time to compare the different detector behaviors. ECRH-modulation experiments have 
been performed for investigating the power deposition and studying heat transport in $\mathrm{He}$ discharges. A 16-channel Si-diode array demonstrates its capability to monitor the heat wave propagation in the plasma due to its higher temporal resolution $(14 \mu \mathrm{s})$. A 12-channel Au-foil bolometer demonstrates higher accuracy in comparison with the Si-diode bolometer for measuring the total plasma radiation because of its broader spectral response covering the wavelength range from soft X-ray down to ultraviolet (UV). The major radiation spectra of the He-plasma at WEGA, estimated based on the Atomic Data and Analysis Structure (ADAS) [8], are in the UV range. Its results are therefore used for the steady-state power balance analysis. However, its time resolution of about $5 \mathrm{~ms}$ is not high enough for monitoring the ECRH-modulation experiments at modulation frequencies of up to $746 \mathrm{~Hz}$.

The paper is organized as follows: The experimental set-up and the experiments are described in section 2. Data analysis of the diode array signals is presented in section 3 . Numerical simulations of the experiment and the determination of the perturbative thermal diffusivity are demonstrated in section 4. Subsequently, in section 5 we briefly show a local power balance calculation using radiated power emissivity profiles measured by the $\mathrm{Au}$ resistive bolometer during the stationary phase of the discharges, through which the steadystate thermal diffusivity is derived. Conclusion remarks and discussions are given in the last section.

\section{Experiment}

The ECRH-modulation experiments for studying the heat transport at WEGA are performed in He-discharges. The plasma is heated by a $28 \mathrm{GHz}$ gyrotron of $9 \mathrm{~kW}$ heating power in total and based on an OXB-heating process, which is realized through a two-step mode conversion, i.e. from electromagnetic ordinary (O)-mode to extraordinary $(\mathrm{X})$-mode and then to an electrostatic Bernstein-wave (B) [9]. The Bernstein waves propagate towards the plasma center, where they are completely absorbed under the resonance condition for the second harmonic. Over-dense plasma with an electron density $n_{e}$ beyond the density cut-off $\left(n_{e, \text { cut-off }}=0.97 \times 10^{19} \mathrm{~m}^{-3}\right.$ for O-mode) can be then achieved. Ray tracing calculations demonstrate that a peaked power deposition profile with a width of $2 \mathrm{~cm}$ could be obtained by

Fig. 1. The Poincaré plot of the closed magnetic flux surfaces confining the plasma, the ECRH power deposition region around the magnetic axis $\left(r_{\text {heat }}=1.0 \mathrm{~cm}\right)$ and the line of sight (LoS) of the 16-channel Si-diode array with solid lines denoting the central line of sight (LoS) and a pair of dashed lines the LoS width of channel 9 (ch9).

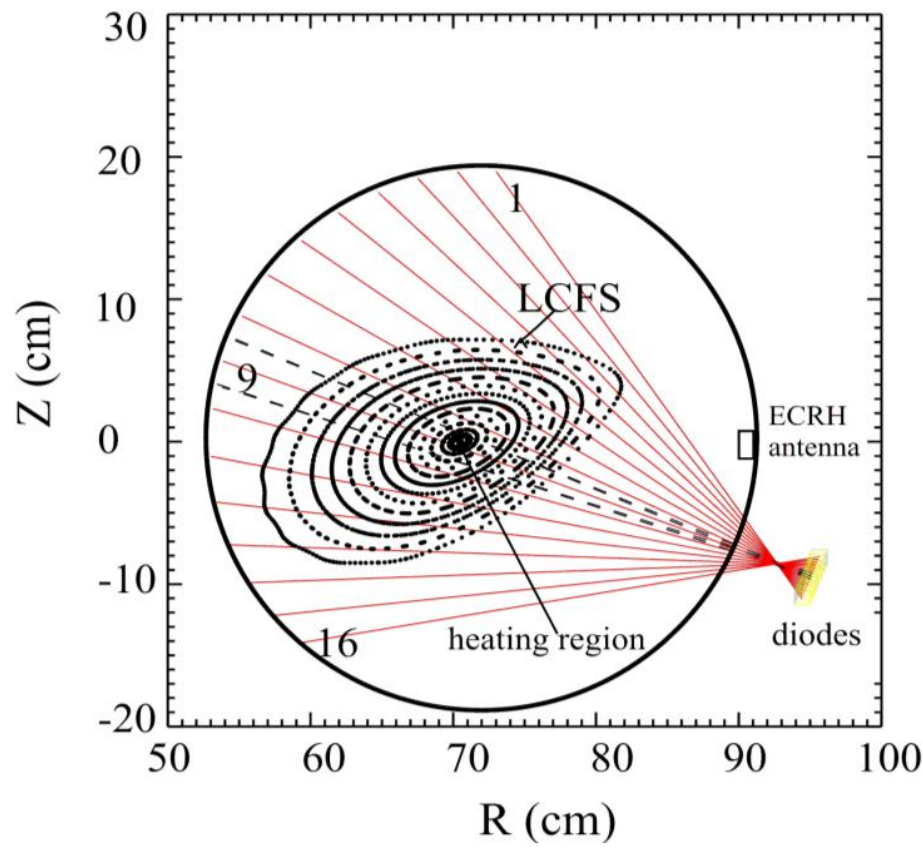


an on-axis resonant heating of the $28 \mathrm{GHz}$ Bernstein waves; a Doppler down shift in the second harmonic absorption should be $0.03 \mathrm{~T}$. The latter has been reproduced experimentally by a magnetic field scan $\left(B_{0}=0.46 \mathrm{~T}-0.5 \mathrm{~T}\right)$; an optimized heating efficiency has been obtained for an on-axis heating with a B-field of $0.47 \mathrm{~T}$ (instead of $0.5 \mathrm{~T}$ without Doppler shift). The ECRH-modulation experiments are carried out under this condition. The actual power deposition corresponds to the cross section of a magnetic flux surface having a circleequivalent area of $\pi r_{\text {eff }}{ }^{2}$ with an effective radius $r_{\text {eff }} \sim 1.0 \mathrm{~cm}$. The Si-diode array having 16 linearly arranged detectors is installed in a port viewing the plasma through a slit-aperture (2 $x 10 \mathrm{~mm}^{2}$ ) from the magnetic low-field side. Its poloidal viewing angle opens over $64^{\circ}$ and covers the whole plasma cross section, including the confined plasma inside the last closed flux surface (LCFS) as well as the edge plasma in the scrape-off layer (SOL). The radial resolution of the diode array at the plasma center is around $3 \mathrm{~cm}$. Fig. 1 illustrates the line of sight (LoS) of the diode-array, its intersection with the closed magnetic flux surfaces (i.e. the Poincaré plot) as well as the heating region, which lies in the LoS of channel 9 (ch9). The radial position of each LoS is denoted by the effective radius $r_{\text {eff }}$ of the closed magnetic surface to which it is tangent.

The OXB-heated He-plasma has a peaked $n_{e}$ profile with a central density around $1.3 \times 10^{19} \mathrm{~m}^{-3}$ and a peaked $T_{e}$ profile with a central $T_{e}$ around $15 \mathrm{eV}$ as shown in Fig. 2 (left). They are measured by a Langmuir probe when the plasma reaches a stationary state. The plasma emissivity profile is highly peaked in this case, documented by Abel-inversion on the line-integrals measured by the Au-resistive bolometer as shown in Fig. 2 (right). It is noteworthy that only the Au-resistive bolometer could be used for measuring the radiative power loss. Because of aging effects [10], the sensitivity of the diode array did not correspond to the calibration in the relevant wavelength range.
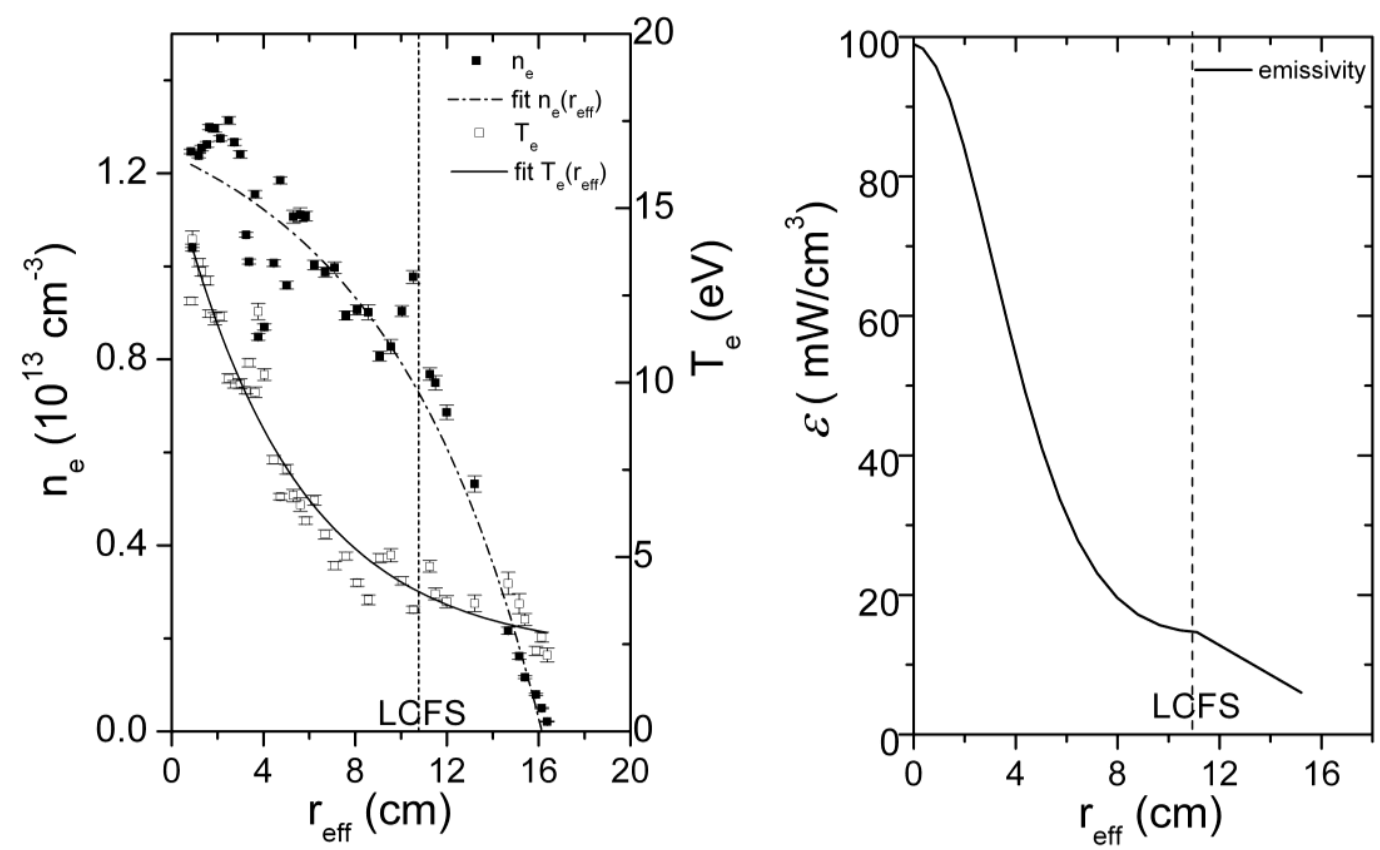

Fig. 2. (Left) $T_{e}$ and $n_{e}$ profile of the OXB-heated He-plasma measured by a Langmuir probe. The dashed and solid lines denote the exponential decay fits to the measured electron densities and temperatures, respectively. (Right) The emissivity profile of the He-plasma obtained through performing Abel-inversion on the line-integrals measured by an Au-resistive bolometer. 
The ECRH-modulation experiments are carried out when the OXB-heated plasma reaches a stationary state. The heating power amplitude is then square-wave modulated within $20 \%$. The modulation frequency $f_{m}$ is restricted to the range from $37 \mathrm{~Hz}$ to $746 \mathrm{~Hz}$. The
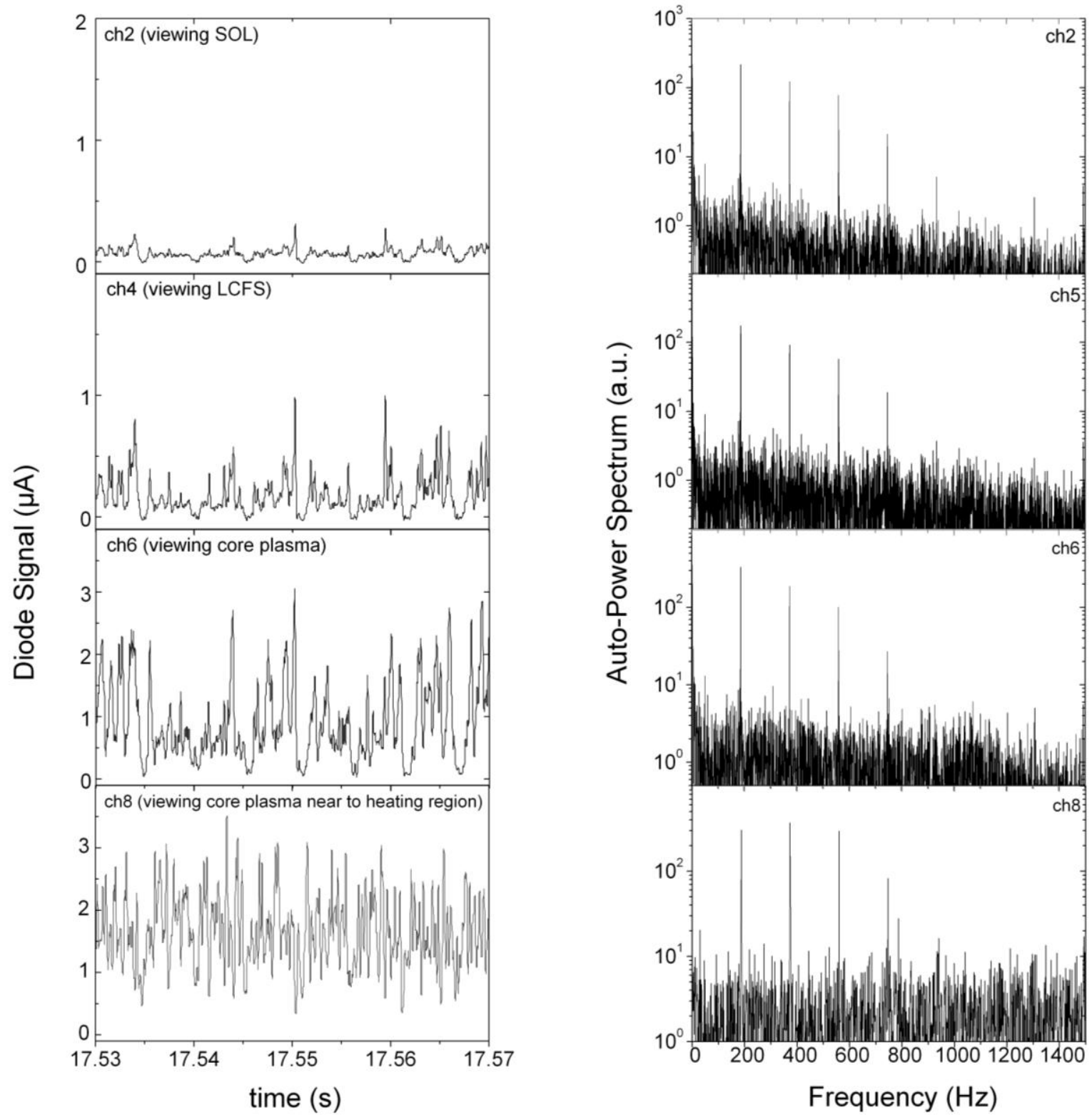

Fig. 3. (Left) Diode signals during the ECRH power modulation experiment for $f_{m}=186 \mathrm{~Hz}$ and duty cycle $=84 \%$ (shot \#33698) and (right) the auto-power spectra of the corresponding channel signals.

plasma density is controlled by keeping the neutral pressure constant $\left(2 \times 10^{-4} \mathrm{mbar}\right)$. All the diode channels are recorded simultaneously with a sampling frequency of $70 \mathrm{kHz}$. Fig. 3 (left) shows the recorded diode signals of different channels, where $f_{m}$ is $186 \mathrm{~Hz}$. FFT algorithms have been performed on the data. The auto-power spectra are shown in the right column of Fig. 3 indicating evident amplitudes of the first three harmonics of $f_{m}$. It is noted that all the measured signals are accompanied by fluctuations with increasing magnitude towards the plasma center. It is not the purpose of this paper to identify and analyze the involved mode activities or instabilities. Since the intensities of the background fluctuation 
spectra as shown in Fig. 3 (right) are around two orders of magnitude smaller than the first three harmonics, their influence to the heat wave propagation induced by ECRH-modulation are negligible. Hereby no efforts have been made to identify the background fluctuations.

\section{Data analysis}

Modulation of the ECR-heating power leads to firstly temperature perturbations in the power deposition region, i.e. the plasma center, and subsequently to a change of the local emissivity. The radial propagation of the temperature perturbation results in a change of the plasma radiation in the entire confined plasma. In view of the peaked emissivity profile (see Fig. 2) as well as the high sensitivity of the local emissivity to the electron temperature (see section 4) the inner plasma zones lying in the corresponding LoS contribute predominately to the line-integrals of the bolometer signal. We select the signal of ch9 as reference for further data analysis since its major component has the same phase as that of the heating power modulation. The ECRH input signal measured with lower sampling rate is not recorded simultaneously and hence is not available as reference.

Based on the cross-correlation technique a cross-correlation function in time-domain is defined as [11]

$$
R_{i j}(\tau)=\left(\frac{1}{t_{m}}\right) \int_{0}^{t_{m}} S_{i}(t) \cdot S_{j}(t+\tau) d t
$$

where $t_{m}$ is the time duration of the experiment and $S_{i}$ and $S_{j}$ are two different signals. Applying the FFT algorithm on $R_{i j}(\tau)$ yields a complex function $G_{i j}=F F T\left\{R_{i j}(\tau)\right\}=$ $\left(F F T\left\{S_{i}\right\}\right)^{*} \cdot F F T\left\{S_{j}\right\}$, with its amplitude $\left|G_{i j}\right|$ and its phase angle standing for the crossspectral power density and the relative phase shift between $S_{i}$ and $S_{j}$, respectively. The star here denotes complex conjugation. Coherence $\gamma_{i j}^{2}$ is defined as the normalized cross power, i.e. $\gamma_{i j}^{2} \equiv \frac{\left|G_{i j}\right|^{2}}{G_{i} G_{j}}$, where $G_{i}, G_{j}$ are the auto-power spectral densities. In the following $G_{i j}$ is calculated in the frequency-domain based on the results of $\operatorname{FFT}\left\{S_{j}\right\}(j=1, \ldots, 16)$ and $\left(F F T\left\{S_{i}\right\}\right)^{*}(i=9)$. Phase delays of the channel signals, shown later in Fig. 6, are the phase shifts relative to the reference $(i=9)$. The coherence is obtained by averaging the $\gamma_{i j}^{2}$ derived from 10 data segments for noise suppression.

The coherence spectra, taking the case of $f_{m}=373 \mathrm{~Hz}$ as example, are shown in Fig. 4. Signal detections at the fundamental frequency as well as at the lower order of harmonics are confirmed accordingly. The associated coherence distribution for the fundamental component versus the diode number $(n c h)$ is depicted in Fig. 5. It is natural that $\gamma^{2}=1$ for $n c h=9$, standing for a total correlation. The coherence amplitude attenuates for the channels with LoS lying outside the heating region. Whereas anomalously high coherences occur for channel 3 and 13, which view the zone near to the LCFS, and for the edge channels ( $n c h=1,2,14,15$, 16), which view the plasma in the SOL (see Fig. 1). This phenomenon, also observed in the experiments with other $f_{m}$, is explained by edge region microwave stray radiation, not 
penetrating into the plasma center and therefore not experiencing OXB mode conversion, hence heating the SOL plasma (in O- or X-mode) more directly through multi-pass absorption processes. The response of these channels to the ECRH-modulation has thus less correlation with the temperature perturbation propagation originating from the plasma center.
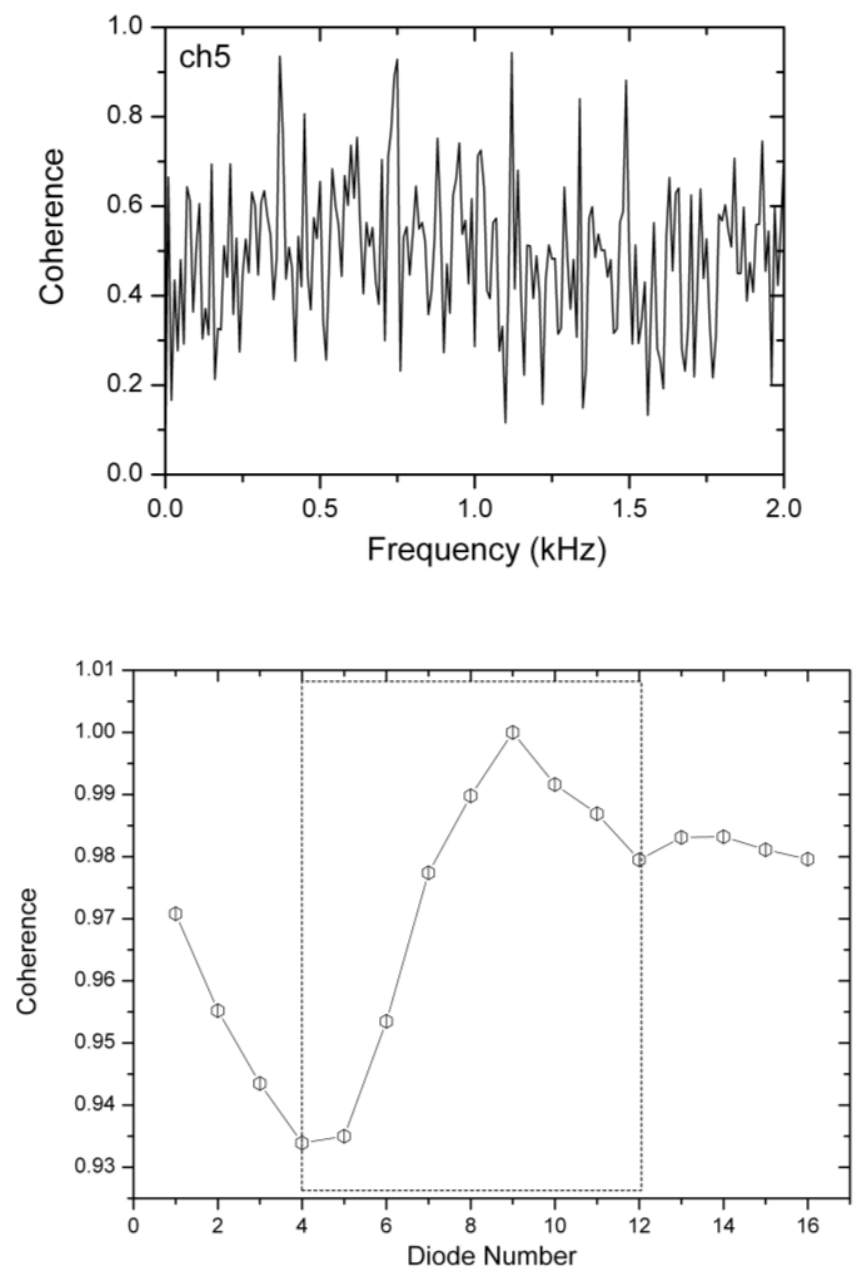

Fig. 4. Coherence spectra between ch5 and the reference ch9 obtained through averaging over 10 time segments of the experiment $\left(t_{m}=1.0\right.$ s). Signal detection at the fundamental frequency $\left(f_{m}=373 \mathrm{~Hz}\right)$ as well as at the lower order of harmonics is confirmed.
Fig. 5. Coherence distribution of the diode signals at the fundamental frequency for $f_{m}=373 \mathrm{~Hz}$ extracted from the coherence spectra. Anomalous coherences of edge channels (nch $<4$ and nch > 12) are explained by the heating of the edge plasma region through microwave stray radiation .

Phase delays $\varphi$ of the fundamental components have been extracted from the phase spectra of function $G_{i j}$ and are shown in Fig. 6. The results for lower $f_{m}(37 \mathrm{~Hz}$ and $93 \mathrm{~Hz})$ are more or less buried in noise and will not be further considered. Additionally, the edge channels with LoS lying beyond or on the LCFS are also not taken into account. The reason is discussed above. On the right part of Fig. 6, the phase delays are plotted as a function of the radial position of the LoS. Estimation of the electron thermal diffusivity is based on this data set. 

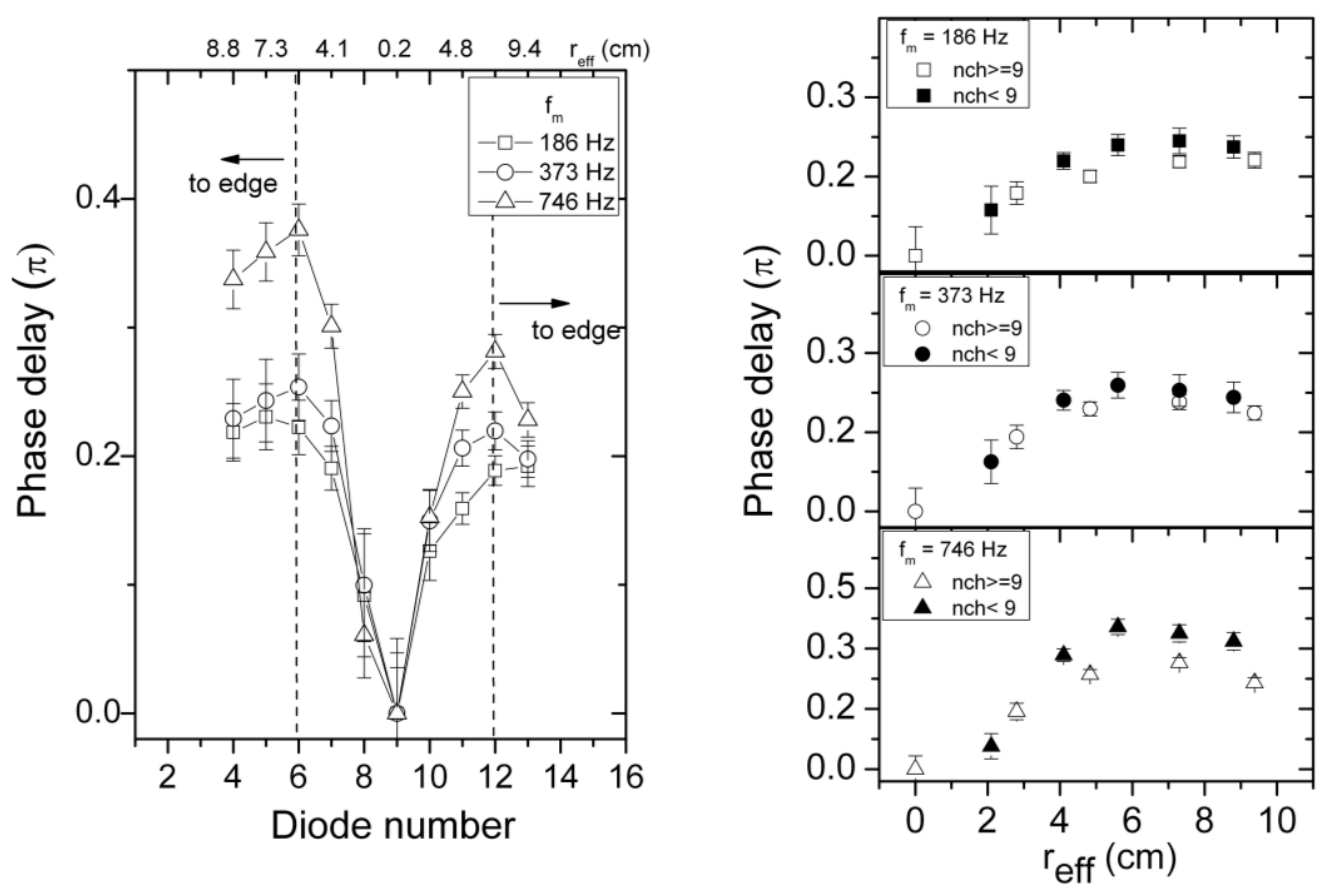

Fig. 6. Phase delays of the fundamental frequency component of the diode signals (left) and its distribution versus the radial position of the LoS (right) for three different ECRH-modulation frequencies $\left(f_{m}=186 \mathrm{~Hz}, 373 \mathrm{~Hz}, 746 \mathrm{~Hz}\right)$; The solid and hollow symbols denote the results of channels whose LoS are on the upper-right (nch <9) and on the lower-left side $(n c h \geq 9)$ of the magnetic axis, respectively.

The line-integrated signal measured by bolometer can be written as the sum of the plasma radiation originating from discrete zones lying in the LoS, i.e. $S=\sum_{j} S_{j}(j=$ $n r_{i}, \ldots, n r_{o}$ ), where $n r_{i}$ and $n r_{o}$ labels the inner most and the outer most zone for an individual LoS, respectively. These zones are ring-shaped; similar with those shown in Fig. 1 defined by the intersection of the LoS set with the closed magnetic flux surfaces. Each of them has a circle-equivalent area and an effective radius. The closed magnetic flux surface, to which the side-LoS of ch9 is tangent, is defined as the first zone. It has an effective radius of $r(1)=1.5$ $\mathrm{cm}$, thus contains the entire heating region $\left(r_{\text {heat }} \approx 1.0 \mathrm{~cm}\right)$. The outermost zone labeled as $n r_{o}$ is surrounded by the LCFS. The zones, beyond the first one, have widths equivalent to $\Delta r=$ $\left(r_{L C F S^{-}} r(1)\right) /\left(n r_{o}-1\right)$, where $r_{L C F S}$ is the minor plasma radius $(10.7 \mathrm{~cm})$. The total zone number, $n r_{o}$, is adjusted that $\Delta r$ is close to the averaged LoS width. We assume that in each zone the plasma parameters and the local emissivity $\varepsilon_{j}$ are constant. The contribution of each zone to the channel line-integral is expressed as $S_{j}=k_{i j} \varepsilon_{j}$, with $k_{i j}$ being the geometric matrix of channel $i$ associated with the magnetic configuration as well as the individual LoS geometry. The response of the line-integral to temperature perturbation can then be expressed as

$$
\tilde{S}=\sum_{n r_{i}}^{n r_{o}} \tilde{S}_{j} \quad\left(j=n r_{i}, \ldots, n r_{o}\right)
$$


where $\tilde{S}_{j}=k_{i j} \widetilde{\varepsilon_{j}}\left(r_{e f f}\right)$, with $\widetilde{\varepsilon_{j}}$ being the local emissivity change induced by the local temperature perturbation $\widetilde{T}$. The relationship of $\tilde{S}_{j}$ to $\widetilde{T}$ is expressed with the help of a Taylor series as

$$
\tilde{S}_{j}=S^{\prime} \cdot \tilde{T}(j)+S^{\prime \prime} \cdot \tilde{T}(j)^{2}+\ldots
$$

where $S^{\prime}=\left.\frac{d S_{j}}{d T_{e}}\right|_{T_{e, j}}$ and $S^{\prime \prime}=\left.\frac{1}{2} \frac{d^{2} S_{j}}{d T_{e}^{2}}\right|_{T_{e, j}}$, respectively.

Under experimental conditions $\left(T_{e, j}=4-15 \mathrm{eV}\right)$, the emissivity $\varepsilon_{j}(r)$ is dominated by the line radiation from $\mathrm{He}^{1+}$. It is a function of the electron density and the temperature expressed approximately as $\varepsilon_{j}(r)=n_{e}^{2}(r) * f\left(T_{e}(r)\right)$, with $f$ representing the line radiation coefficient. In the temperature range involved $f$ increases monotonously with increasing temperature and has a high sensitivity to it $\left(f \sim T_{e}{ }^{\alpha}, \alpha>4\right.$ for $\left.T_{e}<15 \mathrm{eV}\right)$ [8]. According to this estimation, the square term in eq. (3) is smaller than the linear term by a factor of about 7 . Hence in first approximation only the first term is considered. Eq. (3) is rewritten as

$$
\tilde{S}=\sum_{n r_{i}}^{n r_{o}} k_{i j} n_{e}^{2}(r) \frac{d f}{d T_{e}} \tilde{T}(j),
$$

which establishes the basis for the simulation of the experiments.

Eq. (4) demonstrates the smearing-out effect of the line-integrated signal. Considering a harmonic perturbation $\tilde{T}(j)=A(j) e^{i(\omega t+\varphi(j))}$ and the resultant line-integral response, $\tilde{S}=A_{S} e^{i\left(\omega t+\varphi_{S}\right)}$, we understand that the phase angle $\varphi_{S}$ is influenced by the phase delays in all of the viewed zones, $\varphi(j)$, in combination with a weighting factor, i.e. $k_{i j} n_{e}^{2}(r) \frac{d f}{d T_{e}} A(j)$. For the special case that the contribution by the innermost zone dominates the whole signal, the relation of $\tilde{S}$ and $\tilde{T}$ can be simplified as

$$
\tilde{S}=k_{i, n r_{i}} n_{e}^{2}(r) \frac{d f}{d T}(r) \tilde{T}(r)
$$

indicating a direct correlation of the channel signal with a local temperature perturbation. Phase delay distribution derived from the channel signals can then be used for evaluating the thermal diffusivity $D$ according to the following equation $[2,12]$

$$
D=\frac{3 \omega}{4\left(\varphi^{\prime}\right)^{2}}
$$

This analytical solution is deduced from the electron thermal transport equation for simplified slab-geometry.

Numerical simulations in a cylindrical geometry based on eq. (4) are performed in the following section.

\subsection{Heat wave propagation simulation}


The electron energy transport equation in case of a power perturbation $\tilde{S}_{t}$ is given by

$$
\frac{3}{2} n_{e} \frac{d \tilde{T}}{d t}+\nabla \cdot \tilde{q}=\tilde{S}_{e f f}
$$

where $\tilde{q}=-n_{e} x \nabla \tilde{T}+n_{e} v \tilde{T}$ with $x$ being the thermal conductivity and $v$ representing a convective contribution, and $\tilde{S}_{e f f}=\tilde{S}_{t}-n_{e} \cdot \frac{\tilde{T}}{\tau_{e f f}}$ with the second term standing for all perturbed sinks proportional to $\tilde{T}$, e.g. electron-ion energy exchange through elastic collisions and radiation losses [12]. Since the second term functions actually as a damping term, it has an effect on the amplitude change of $\widetilde{T}$ and less effects on the phase. For the sake of simplicity to perform the simulations of phase delays, $\tilde{S}_{e f f}$ is taken as $\tilde{S}_{t}$. Let $\tilde{E}=\frac{3}{2} n_{e} \tilde{T}$ and $D=\frac{2}{3} x$. Then Eq. (7) becomes

$$
\frac{d \tilde{E}}{d t}+\nabla \cdot\left[-\nabla D \tilde{E}+\left(D \frac{\nabla n_{e}}{n_{e}}+\frac{2}{3} v\right) \tilde{E}\right]=\widetilde{S}_{t}
$$

We are interested only in the radial transport. Furthermore, we assume $v=0$ and that the flux surfaces are nested circles. In this case, Eq. (8) becomes one dimensional and can be solved using different numerical methods [2, 4, 12]. Here, we present a Monte Carlo method for its numerical simplicity. Especially in view of the complex geometries involved, Monte Carlo integration demonstrates its evident advantages [11]. "Particles" representing in our case a certain amount of energy are started from the power deposition zone and weighted by $\tilde{S}_{t}$. They are traced in Cartesian coordinates as

$$
\begin{aligned}
& x_{t+\tau}=x_{t}+\xi_{x} \sqrt{2 D \tau}+v_{x}^{*} \tau, \\
& y_{t+\tau}=y_{t}+\xi_{y} \sqrt{2 D \tau}+v_{y}^{*} \tau,
\end{aligned}
$$

where $\left[x_{t}, y_{t}\right]$ is the location of a Monte Carlo "particle" at the time $t$ and $\left[x_{t+\tau}, y_{t+\tau}\right]$ is the termination after a small time step $\tau$. $\xi_{x}$ and $\xi_{y}$ are two independent random numbers taking the value of \pm 1 with equal probability of $50 \%$. $v_{x}^{*}$ and $v_{y}^{*}$ are the $x$ and $y$ components of $D \frac{\nabla n_{e}}{n_{e}}$. Then, at any later time point $t, \tilde{E}$ is given by summing up all the Monte Carlo particles enclosed in the volume $\Delta V$, i.e.

$$
\tilde{E}(x, y, t)=\frac{\sum_{i=1}^{N} w_{i}}{\Delta V}, \quad \text { with } w_{i}=\tau \frac{\widetilde{s_{t}}\left(t-n_{i} \tau\right)}{N_{\tau}} \text { and }\left(x_{i}, y_{i}\right) \in \Delta V
$$

where $n_{i}$ is the number of steps tracing particle $i$ so far, $N_{\tau}$ is the number of Monte Carlo particles generated within each time step $\tau$ and $N=N_{\tau} t \tau$ is the total number of particles generated up to time point $t$.

Proper selection of $\tau$ and $N_{\tau}$ is necessary in order to reduce the calculation error. In general, radial amplitude of the random walking step $\Delta r_{\max }=\sqrt{4 D \tau}$ should be much smaller than the zone width, which is close to the averaged LoS width. $N_{\tau}$ should be large enough in order to reduce numerical errors. Reducing $\tau$ and increasing $N_{\tau}$ are therefore favorable, which 
are, however, limited by the computing time. We employ $\tau=5 \mu \mathrm{s}$ and $N_{\tau}=800$ for our calculation.

\subsection{Line-integral simulation}

The simulations are carried out for different modulation frequencies while keeping the duty cycle at $84 \%$ as in the experiment. The finite power deposition region around the magnetic axis is also taken into account, which, in the particle picture above, corresponds to the initial position of "particle" generations.

The line-integrated signals are simulated according to Eq. (4). The temperature perturbation propagation $\tilde{T}(r, t)$ is reflected by $\tilde{E}(x, y, t)$ resolved above, assuming the electron density $n_{e}$ unchanged during ECRH-modulation experiment. The zone radiation sensitivities to temperature perturbations $S^{\prime}(r)=\left.k_{i j} n_{e}^{2}(r) \frac{d f}{d T}\right|_{T_{0, j}}$ has been evaluated according to the $n_{e}(r)$ and $T_{e}(r)$ profile (see Fig. 2) as well as the line-radiation coefficient $f$ of $\mathrm{He}^{1+}$ in ADAS [8]. They are adopted as weighting factors for the particles loaded in zones during performing Monte Carlo integrations for an individual LoS.

Data analyses of the calculated line-integrals are subsequently performed with the same method as dealing with the measured signal, i.e. the cross-correlation technique in the frequency domain. Ch9 viewing the heating region is again taken as the reference. Phase delays of the simulated line-integrals for the fundamental modulation frequencies are then obtained, which is a function of the input thermal diffusivity $D$. The line-integral amplitude itself is normalized and not further used.

\subsection{Determination of the thermal diffusivity coefficient}

The heat diffusivity is determined through matching the calculated phase delay profile to the experimental results. Electron thermal diffusivities of $1.5 \mathrm{~m}^{2} / \mathrm{s}, 2.0 \mathrm{~m}^{2} / \mathrm{s}$ and $2.2 \mathrm{~m}^{2} / \mathrm{s}$ are obtained for $f_{m}=186 \mathrm{~Hz}, 373 \mathrm{~Hz}$ and $746 \mathrm{~Hz}$, respectively, through minimizing the averaged deviation between the calculated and the measured data. Fig. 7 illustrates the results, taking into account the channels viewing the central plasma region. An averaged perturbative thermal diffusivity of $D=1.9 \mathrm{~m}^{2} / \mathrm{s}$ with an uncertainty of $\pm 0.4 \mathrm{~m}^{2} / \mathrm{s}$ is obtained. In order to show the sensitivity of this method, phase delays calculated for higher diffusivitiy $(D=5.0$ $\mathrm{m}^{2} / \mathrm{s}$ ) are also plotted in Fig. 7. The discrepancy of the $D$ values for different $f_{m}$ may be due to experiment errors. To confirm the frequency-dependency of the perturbative thermal diffusivity, it is necessary to increase the number of data points through prolonging the experiment duration and to improve the spatial resolution of the diode array.

If the contributions from outer zones in the $\operatorname{LoS}$ are ignored, estimation in slabgeometry based on Eq. (6) can be done. Utilizing the phase delay derivatives, $\varphi^{\prime}=0.13 \mathrm{~cm}^{-1}$, $0.14 \mathrm{~cm}^{-1}$, and $0.21 \mathrm{~cm}^{-1}$ according to the experimental data for $f_{m}=186 \mathrm{~Hz}, 373 \mathrm{~Hz}$ and 746 $\mathrm{Hz}$, respectively, in Fig. 6 (right) yields an averaged thermal diffusivity $D=8.0 \mathrm{~m}^{2} / \mathrm{s}$. This 
result is much higher (around 4 times) than that from the simulation, showing that the assumption of a slab-geometry is not correct.

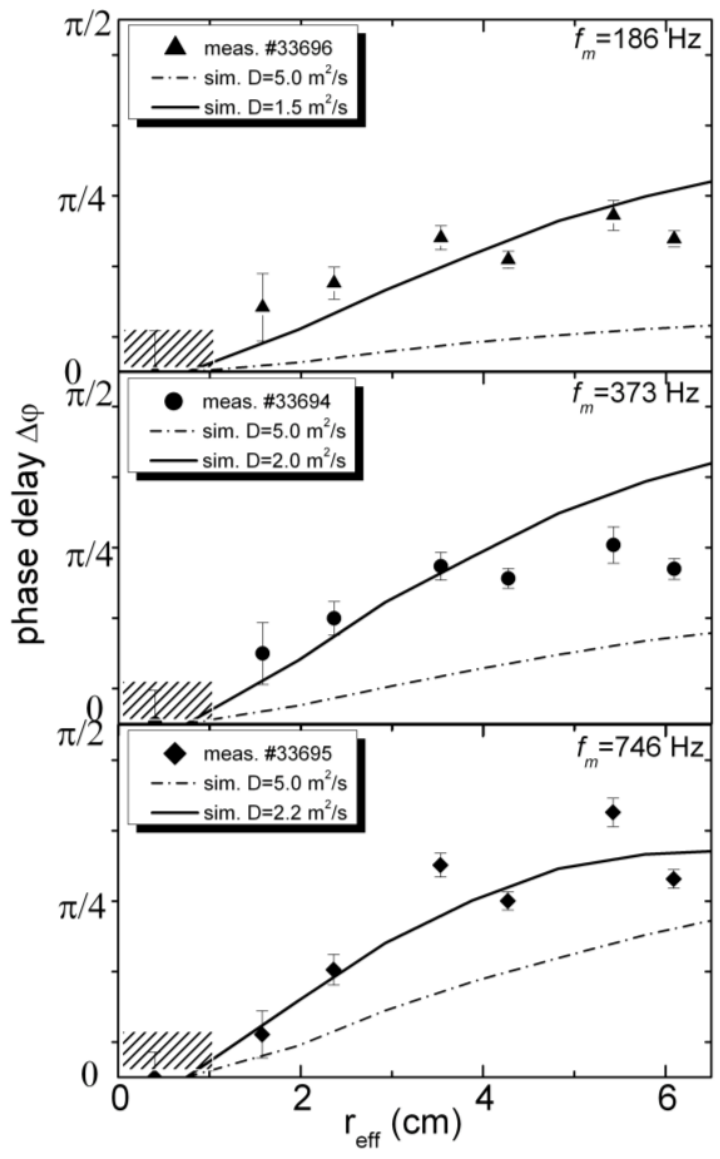

Fig. 7. Determination of the electron thermal diffusivity $D$ through matching the simulated phase delays (solid lines) to the experimental data (symbols). An averaged thermal diffusivity of $D=1.9 \pm 0.4 \mathrm{~m}^{2} / \mathrm{s}$ is obtained for the central plasma. The dotteddashed lines illustrate the calculated phase delays for $D=5.0 \mathrm{~m}^{2} / \mathrm{s}$, demonstrating the sensitivity of the algorithm used. The dashed area indicates the heating region.

\section{Evaluation of the steady-state thermal diffusivity}

In this section the steady-state thermal diffusivity $D^{P B}$ is evaluated from a local power balance calculation within the LCFS for the same OXB-heated He-plasma described in section 2 and compared with the one obtained in the last section. The relation or the difference of them reflects the plasma response onto perturbations $[4,15]$. The power balance reads as

$$
P_{\text {heat }}=P_{\text {trans }}+P_{\text {rad }}
$$

where $P_{\text {heat }}$ is the ECR-heating power deposited in a central plasma region enclosed in an effective radius $r_{\text {eff }}, P_{\text {rad }}$ is the radiative power loss and $P_{\text {trans }}$ is the power transported out of this region, perpendicular to its magnetic flux surface, respectively. Assuming diffusive processes for energy and particles, the heat flux across the $r_{\text {eff }}$ surface can be written as [13]

$$
\frac{P_{\text {trans }}}{A}=-n_{e} D^{P B} \frac{\partial T_{e}}{\partial r}-\frac{5}{2} T_{e}\left(D_{P} \frac{\partial n_{e}}{\partial r}+n_{e} v\right),
$$


where $A$ is the surface area, $D^{P B}$ is the radial thermal diffusivity to be evaluated, $D_{P}$ is the radial particle diffusivity and $v$ is the particle convection velocity, respectively. The ions in the WEGA plasmas are cold $(<1 \mathrm{eV})$ and their contribution to the power flow is not considered.

A local power balance is made for the central plasma region inside the $r_{\text {eff }}=6.5 \mathrm{~cm}$ surface, $\rho=r_{e f f} / r_{L C F S}=0.6$, which corresponds the region discussed in the last sections for the ECRH-modulation experiments. According to the 1D emissivity profile shown in Fig. 2, the radiated power loss in the region is evaluated as $P_{\text {rad }}=4 \pi^{2} R \int_{0}^{r} e f f \varepsilon(r) r d r=3.7 \mathrm{~kW}$. Assuming that the absorbed heating power in form of Bernstein-wave, $P_{\text {heat }}$, is $70 \%$ of the total input ECRH power $(7.6 \mathrm{~kW})$ indicated by a microwave detector, a sniffer probe, which measures the non-absorbed power level during the experiment and monitors the drop of the reflected microwave during the transition into Bernstein-wave heating, the radial power flux across the $r_{\text {eff }}$ surface, $P_{\text {trans }}$, is thus obtained.

In the further calculation, the particle pinch term in Eq. (13) is ignored and the particle diffusivity coefficient is taken as $D_{P}=3.8 \mathrm{~m}^{2} / \mathrm{s}$ determined by a particle balance analysis performed for the He-plasma [14]. There, a radially independent $D_{P}$ is assumed and particle convection is ignored. The sink and source terms associated with the atomic processes, including collisional dielectronic ionization of $\mathrm{He}$ and $\mathrm{He}^{1+}$ as well as recombination of $\mathrm{He}^{1+}$ and $\mathrm{He}^{2+}$ are taken into account. The rate coefficients of the processes are adopted from ADAS [8]. $D_{P}$ is determined through matching the calculated $n_{e}$ profile to the measured one.

The electron temperature gradient $\frac{\partial T_{e}}{\partial r}$ and the density gradient $\frac{\partial n_{e}}{\partial r}$ at $r_{\text {eff }}=6.5 \mathrm{~cm}$ in Eq. (13) are obtained through exponential fits of the measured data plotted in Fig. 2 (left). A local thermal diffusivity of $D^{P B}=3.5 \mathrm{~m}^{2} / \mathrm{s}$ is then obtained. A radial diffusivity profile $D^{P B}(r)$ is potentially obtainable by shifting $r_{\text {eff }}$ outward and inward. Estimation for an inner region indicates higher diffusivities. A detailed error analysis indicates that the uncertainty involved in the local power balance calculation is evident and predominantly due to the uncertainties of the absorbed heating power $(\sim 15 \%)$, the radiated power derived from Abelinverted emissivity profile $(\sim 15 \%)$ and also the plasma parameters $n_{e}$ and $T_{e}(\sim 5 \%)$ as well as their gradients $(\sim 13 \%)$. A relative error of the deduced $D^{P B}$ is estimated as high as $90 \%$ taking into account all the above factors, including the error in determining $D_{P}$ in Ref. [14], which is around $30 \%$. Improved knowledge about the absorbed heating power and higher accuracies of the involved diagnostics, bolometer and Langmuir probe, are required for improving the local power balance calculation. The radial diffusivity profile is therefore not discussed in this paper.

\section{Conclusion remarks and discussions}

Conclusion remarks: Bolometers measure the line-integrated plasma radiation. The sensitivity of the bolometer signal to an electron temperature perturbation is associated with the temperature dependence of the emissivities along the line of sight. In certain $T_{e}$ ranges the sensitivity of the emissivity to $T_{e}$ can be much higher than that to $n_{e}$. This is the case for the 
WEGA plasmas which have electron temperatures typically between 4 and $15 \mathrm{eV}$, therefore making it possible to use the bolometer for analyzing heat transport.

Density effects are ignored in our analysis for the following reasons: First, the modulated ECRH-power introduces primarily a $T_{e}$ perturbation. Second, the modulation magnitude is kept small (20\%) and the modulation duty cycle is $84 \%$. At last, the modulation period is from $1.3 \mathrm{~ms}$ up to $5.6 \mathrm{~ms}$ ( for $f_{m}=746 \mathrm{~Hz}-176 \mathrm{~Hz}$ ) and the ECRH-off-time in each modulation-period is $0.2 \mathrm{~ms}$ up to $0.9 \mathrm{~ms}$, which is much shorter than the estimated particle confinement time at WEGA $(7 \mathrm{~ms})$. During the ECRH-modulation experiment, the lineintegrated electron density measured by interferometer showed a relative change of about $5 \%$, confirming that the electron density $n_{e}$ change is minimal.

The present work provides a simple method of estimating the perturbative electron thermal transport coefficient based on a fast bolometer diode diagnostic. This is the only diagnostic currently available at WEGA for monitoring the heat transport process induced by ECRH-modulation. The bolometer results confirm the centrally localized power deposition and the outward heat propagation. According to the width of the line of sight of diode 9 at the magnetic axis $(3 \mathrm{~cm})$, the power deposition width derived is $\sim 3 \mathrm{~cm}$. The discrepancy with the ray tracing calculation result $(2 \mathrm{~cm})$ is due to the limited spatial resolution of the bolometer.

The favorable conditions for applying bolometer on perturbative thermal transport experiment for He-discharges are the low $T_{e}$ (around $20 \mathrm{eV}$ ) and the highly peaked emissivity profile in WEGA. The sensitivity of line radiation (from $\mathrm{He}^{1+}$ ) to temperature perturbations reaches the maximum at $T_{e}=20 \mathrm{eV}$ and then drops with further increasing $T_{e}$. For $T_{e} \sim 100$ $\mathrm{eV}$, the sensitivity falls to about $10 \%$ of that at $20 \mathrm{eV}$. The application of the bolometer to heat transport study can be extended to other experiments with different working gases, however, under a requirement of monotonic responses of emissivities to $T_{e}$-perturbation in the studied $T_{e}$-ranges. A special case is as discussed above, $d \varepsilon(r) / d r<0$ (peaked emissivity profile) and $d \varepsilon(r) / d T_{e}>0$ (enhanced emissivity with increment of $T_{e}$ ). Further improvements of the measurement accuracy are possible through refining the spatial and temporal resolution. This can be achieved by increasing the aperture-detector array distance and by rearranging the available channels for the core region only since the edge channels provide no additional information for the heat wave propagation study. Smaller ECRH-modulation amplitude and prolonging the experiment duration (up to $10 \mathrm{~s}$ possible at WEGA) can improve the phase delay accuracy further. Absolute calibrations of the diagnostics are not required on the other hand.

Numerical simulation taking into account the radial dependence of the phase delays of the signal composition (for excluding the smearing-out effect) and the curvature of the magnetic surfaces yields a more accurate result of the thermal diffusivity $\left(D=1.9 \mathrm{~m}^{2} / \mathrm{s}\right)$ than an estimation in slab-geometry. The latter overestimate the thermal diffusivity by a factor of around 4 , indicating the slab-geometry approximation is not applicable.

The local power balance calculation yields a thermal diffusivity of around $3.5 \mathrm{~m}^{2} / \mathrm{s}$ for $\rho=0.6$. The discrepancy between the power balance and the perturbative values is partly related to the uncertainties discussed. Further conclusion about the linearity of the plasma 
response on perturbations in stellarators $[4,15]$ cannot be inferred due to the evident error level in the power balance analysis.

Discussion: According to the electron density and temperature profile shown in Fig. 2 (left), the kinetic energy of the electrons inside $\rho=0.6$ is evaluated as $E=2 \pi R$. $\int_{0}^{\rho}\left(3 T_{e} n_{e} / 2\right) \cdot(2 \pi r) d r=1.6 \mathrm{~J}$. The energy confinement time $\tau_{E, 0.6}$ in the region is then 0.4 $\mathrm{ms}$ according to the formulas $\tau_{E}=E / P_{\text {heat }}$. The characteristic time of the heat diffusivity $\tau_{\text {diff }}$ is estimated as $\tau_{\text {diff }}=r_{\text {eff }}{ }^{2} / 2 D$, where $D$ takes $\sim 2.7 \mathrm{~m}^{2} / \mathrm{s}$ (averaged on the perturbative and power balance value), $\tau_{\text {diff }, 0.6}=0.7 \mathrm{~ms}$ is obtained. They coincide within error bars, confirming that the electron energy transport is indeed a conductive process. The obtained electron thermal diffusivities, $D$ and $D^{P B}$, are clearly above the neoclassical level $\left(\chi_{\mathrm{e}}\right.$ $\sim 0.3 \mathrm{~m}^{2} / \mathrm{s}$ ) [15]. Another argument to exclude the neoclassical transport at WEGA is the low $T_{e}$, conducting to a high collisionality $v_{e i}$ beyond the neoclassical regime. The thermal diffusivities, however, agree well the Bohm-diffusion scaling [16] $\left(\chi_{e}=T_{e} / 16 B \approx 2.0 \mathrm{~m}^{2} / \mathrm{s}\right.$, for $T_{e}=15.0 \mathrm{eV}$ and $B=0.5 \mathrm{~T}$ ). The detailed physical picture of the Bohm-like diffusion associated with plasma microstabilty is still unclear. In literatures $[17,18]$ it is concluded that Bohm-diffusion originates from electric field fluctuations with frequencies in the order of from $\mathrm{kHz}$ up to $\mathrm{MHz}$. In this light, it is interesting for us to study the electric field fluctuations in the central region of the Bernstein-wave heated plasmas and its correlation with the thermal transport processes. And whether the transport process at WEGA is really Bohm-like needs further investigations.

The global energy confinement time within the LCFS $(\rho=1)$ is estimated to be $\tau_{E, 1} \approx 0.6 \mathrm{~ms}$. The heat diffusion time $\tau_{\text {diff,1 }}$ is calculated, based the formulas above, as around $2.0 \mathrm{~ms}$, more than 3 times larger than $\tau_{E, 1}$. This could imply an enhanced heat transport in the outer region beyond $\rho=0.6$. It is interesting to see that the ISS04 stellarator scaling law [19], $\tau_{E}^{I S S 04}=0.134 a^{2.28} R^{0.64} P^{-0.61} \bar{n}_{e}^{0.54} B^{0.84} t_{2 / 3}^{0.41}$, yields a $\tau_{E}$ of $1.7 \mathrm{~ms}$ for the studied plasma $\left(t_{2 / 3}=0.34\right)$ at WEGA.

\section{ACKNOWLEDGMENTS}

The corresponding author gratefully acknowledges the proofreading of this paper by L. Giannone, the fruitful discussions with H. Thomson, Y. Feng, R. Burhenn and R. König and the encouragement of this work by F. Wagner as well as the technical supports by D. Aßmus and M. Marquardt.

\section{REFERENCES}

[1] H.J. Hartfuss, H. Maassberg, M. Tutter, and the W VII-A Team, Nucl. Fusion 26, 678 (1986).

[2] G.L. Jahns, S.K. Wong, R. Prater, S.H. Lin, S. Ejima, Nucl. Fusion 26, 226 (1986). 
[3] L. Giannone, V. Erckmann,et.al., Nuclear Fusion, Vol.32, No. 11,1985 (1992)

[4] N.J. Lopes Cardozo, Plasma Phys. Contr. Fusion 37,799 (1995)

[5] M. Otte et al., Plasma and Fusion Research, Vol. 5, S2011-4 (2010)

[6] K. F. Mast, J. C. Vallet, C. Andelfinger, P. Betzler, H. Kraus, and G. Schramm, Rev. Sci. Instrum. 62, 744 (1991).

[7] R.L. Boivin, J.A. Goetz, E.S. Marmar, J.E. Rice and J.L. Terry, Rev. Sci. Instrum. Vol. 70 (1), 260-264 (1999)

[8] H.P. Summers: Atomic Data and Analysis Structure, JET Joint Undertaking. Report JET-IR(94)06 (1994).

[9] H.P. Laqua et al., Phys. Rev. Lett. 78, 3467 (1997)

[10] G. Veres et al., J. Nucl. Mat. 363-365 (2007) p. 1104-1109

[11] William H. Press, et al., Numerical Receipts: The Art of Scientific Computing, 1989

[12] A. Jacchia and P. Mantica, Phys. Fluids B 3, 3033(1991)

[13] G. D. Porter, Phys. Plasmas 5, 4311 (1998)

[14] D. Zhang et al., "Simulation of the Spectrally-resolved Plasma Radiation in the WEGA Stellarator and Comparison with Bolometer Measurements", 38th EPS Conference on Plasma Physics, Strasbourg (2011), http://ocs.ciemat.es/EPS2011PAP/pdf/PD5.01.pdf

[15] F. Wagner and U. Stroth 1993 Plasma Phys. Control. Fusion 351321

[16] F.F. Chen, Introduction to Plasma Physics and Controlled Fusion, P191, Vol. 1, 2nd ed. (Plenum Press, 1984).

[17] E. Bultinck et al., 2010 J. Phys. D: Appl. Phys. 43292001

[18] E. Martines, R. Cavazzana et al., Phys. Plasmas 83042 (2001)

[19]H. Yamada et al., Nucl. Fusion 45 1684(2005) 